\title{
Ovarian Cystic Teratoma
}

National Cancer Institute

\section{Source}

National Cancer Institute. Ovarian Cystic Teratoma. NCI Thesaurus. Code C7283.

A teratoma that arises from the ovary and is characterized by the presence of cystic structures. Representative example is the dermoid cyst. 